\title{
Genetic Analysis of Transvection Effects Involving cis-Regulatory Elements of the Drosophila Ultrabithorax Gene
}

\author{
José-Luis Micol, ${ }^{1,2}$ James E. Castelli-Gair and Antonio Garcia-Bellido \\ Centro de Biologia Molecular, C.S.I.C.-Universidad Autónoma de Madrid, 28049 Madrid, Spain \\ Received January 20, 1990 \\ Accepted for publication June 8,1990
}

\begin{abstract}
The Ultrabithorax $(U b x)$ gene of Drosophila melanogaster contains two functionally distinguishable regions: the protein-coding Ubx transcription unit and, upstream of it, the transcribed but nonprotein-coding bxd region. Numerous recessive, partial loss-of-function mutations which appear to be regulatory mutations map within the bxd region and within the introns of the Ubx transcription unit. In addition, mutations within the Ubx unit exons are known and most of these behave as null alleles. $U b x^{l}$ is one such allele. We have confirmed that, although the $U b x^{\prime}$ allele does not produce detectable Ubx proteins (UBX), it does retain other genetic functions detectable by their effects on the expression of a paired, homologous $U b x$ allele, i.e., by transvection. We have extended previous analyses made by $\mathrm{E}$. B. Lewis by mapping the critical elements of the $U b x$ gene which participate in transvection effects. Our results show that the $U b x^{l}$ allele retains wild-type functions whose effectiveness can be reduced (1) by additional cis mutations in the bxd region or in introns of the Ubx transcription unit, as well as (2) by rearrangements disturbing pairing between homologous $U b x$ genes. Our results suggest that those remnant functions in $U b x^{I}$ are able to modulate the activity of the allele located in the homologous chromosome. We discuss the normal cis regulatory role of these functions involved in trans interactions between homologous $U b x$ genes, as well as the implications of our results for the current models on transvection.
\end{abstract}

$\mathrm{T}$ HE Ultrabithorax $(U b x)$ gene is one of the selector genes controlling segmental identities in the body of Drosophila melanogaster. Mutations in this gene dramatically alter the cuticular structures of the second thoracic (T2), the third thoracic (T3) and the first abdominal (A1) segments of the adult body of the fly: gain-of-function alleles of the $U b x$ gene transform T2 toward T3 (reviewed in GONZÁLEZ-GAITÁN, MiCOL and GaRCía-Bellido 1990); loss-of-function alleles of the $U b x$ gene give rise to the homeotic transformation of T3 toward T2 and/or that of anterior A1 toward anterior T3 (reviewed in MORATA, SÁNCHEZ-HERRERo and CASANOVA 1986; DunCaN 1987; PeIfer, Karch and Bender 1987; SÁNChezHerrero et al. 1988; MAHAFFeY and KaUfMAN 1988). The $U b x$ gene is composed of three transcription units (see Figure 1): the Ubx unit, coding for mRNAs that are translated into proteins (UBX) with morphogenetic functions, and the overlapping early bxd and late bxd units coding for RNAs of unknown function (Beachy, Helfand and Hogness 1985; Hogness et al. 1985; Lipshitz, Peattie and Hogness 1987; O'CoNNOR et al. 1988). However, mutations in the bxd region ( $b x d$ and $p b x$ partial loss-of-function alleles; BENDER et al. 1983, 1985) affect the spatial

\footnotetext{
'Present address: Division of Biology 156-29. California Institute of Technology, Pasadena, California 91125.

${ }^{2}$ To whom correspondence should be addressed.

pattern of proteins encoded by the Ubx unit, causing absence of UBX in specific regions of embryonic and larval tissues (INGHAM 1985; WhITE and WILCOX 1985; Cabrera, Botas and García-Bellido 1985; Botas, Cabrera and García-Bellido 1988). Mutations affecting introns of the Ubx unit ( $a b x$ and $b x$ partial loss-of-function alleles) also cause local abnormalities in the UBX pattern (BENDER et al. 1983; Peifer and Bender 1986; White and Wilcox 1985; Cabrera, Botas and García-Bellido 1985; Botas, CABRERA and García-Bellido 1988).

Two different classes of apparently null alleles are known in the Ultrabithorax gene: While $a b x, b x$, $p b x$ and $b x d$ recessive alleles inactivate subfunctions of the $U b x$ gene, $U b x$ lack-of-function alleles inactivate most, perhaps all, functions of the gene. $U b x$ lack-offunction alleles as a rule cause the absence of functional proteins in the entire realm of action of the gene and lethality in homozygous embryos (LEWIS 1978; Hayes, Sato and Denell 1984). Ubx lack-offunction alleles include chromosome rearrangements that interrupt the Ubx transcription unit and pseudopoint mutations affecting Ubx unit exons (called pseudopoint as they are not visible in polytene chromosomes but usually correspond to mutational damage greater than a single nucleotide change) (BENDER et al. 1983; HogNess et al. 1985). 


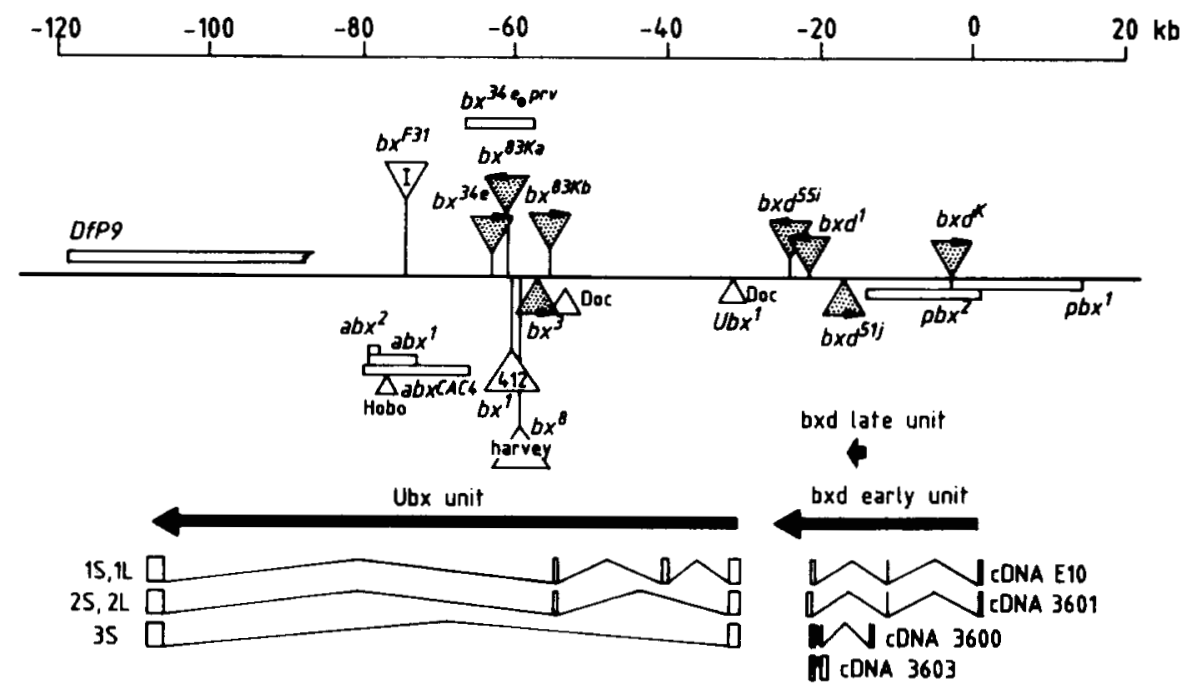

FIGURE 1.- Molecular map of the Ultrabithorax gene showing mutations used in this work. Rectangles indicate deletions and triangles insertions, having the latter the corresponding transposon name $(1,412$, harvey, Hobo and Doc). Dotted triangles are gypsy insertions, the arrow indicating when known the direction of their transcription. $b x^{3}$ is associated with two adjacent insertions (gypsy and Doc), the second being irrelevant for mutant phenotype. abx $x_{i \rightarrow c}^{(i t}$ deletion appeared with a Hobo insertion. $D f(3 R) P 9$ deletes the entire bithorax complex, its left end appearing in the drawing. $D f(3 R) U b \times 109$, which does not appear in the figure, deletes the entire $U b x$ gene and a part of the adjacent $a b d A$ gene, its ends would be out of the drawing. Transcription units are indicated as heavy horizontal arrows. Mature RNAs appear under the transcription units, showing exons as rectangles. Capital letters S and L in Ubx transcription unit mature R NAs indicate short and long, corresponding to the use of either of two alternative splicing donor sites in the 5' exon. All data for this figure were obtained from BENDER et al. 1983, 1985; PeifER and Bender 1986; Lipshitz, PEATTIE and Hogness 1988; O'ConNor et al. 1988; WeinzierL et al. 1987.

Ubx pseudopoint mutant alleles are not true null alleles: $U b x$ pseudopoint mutant alleles show in homozygotes the same phenotypes as null alleles (breakpoints and deficiencies) (LEWIS 1963; MORATA and García-Bellido 1976; KerRIDGE and Morata 1982; Miñana and García-Bellido 1982; Hayes, Sato and Denell 1984). However, $U b x$ pseudopoint mutant alleles are not completely amorphic because their trans heterozygotes with recessive alleles have adult mutant phenotypes less extreme than those observed for those recessives in heterozygotes with deficiencies or breakpoints in the $U b x$ gene (MORATA and KERRIDGE 1980; KerRIDGe and Morata 1982; WeINZIERL $e t$ al. 1987). This inference is supported by observations indicating that $U b x$ pseudopoint alleles behave differently than breakpoints and deficiencies, with respect to sensitivity to ether-induced bithorax phenocopies and to interactions with mutations in the Regulator of bithorax gene (CAPDEvila and GarcíaBellido 1974, 1978, 1981; J. E. Castelli-Gair and A. García-Bellido, manuscript in preparation).

$U b x^{l}$ is a pseudopoint $U b x$ allele lacking detectable protein products: One such pseudopoint $U b x$ allele is $U b x^{l}$ which is associated with the insertion of a Doc transposable element in the untranslated region of the $5^{\prime}$ exon of the Ubx unit (Figure 1) (WEINZIERL et al. 1987). It is not known whether or not this mutation perturbs the transcription of the Ubx unit and/or those of the bxd units. Previous genetic studies and this work show that $U b x^{l}$ mutation in trans-heterozygotes with recessive alleles of the $U b x$ gene give rise to T3 toward T2 homeotic transformations which are less extreme than those observed for the same recessives over a deficiency for the gene (MORATA and KERRIDGE 1980; KerRIDGE and MORATA 1982; WeINZIERL et al. 1987). This behavior could reflect residual activities of some putative protein product of the $U b x^{I}$ allele. However, several observations strongly suggest that the $U b x^{I}$ allele does not produce protein products. First, $U b x^{1}$ homozygous phenotypes are the same as those observed for breakpoints and deficiencies for the gene in mitotic recombination clones in adults (MORATA and GaRCÍA-BELlido 1976; KeRRIDGE and Morata 1982; Miñana and García-Bellido 1982), in gynanders (LEWIS 1963) and in lethal embryos (Lewis 1963, 1978; Hayes, Sato and Denell 1984). Second, $U b x^{1}$ homozygous embryos lack any protein recognizable by FP3.38 (WEINZIERL et al. 1987), an antibody raised against the $5^{\prime}$ exon common to all the mRNAs from the Ubx transcription unit (WHITE and WILCox 1984). Similarly, FP3.38 antibody does not recognize any protein arising from $U b x^{\prime}$ in larval imaginal discs (CASTELLI-GAIR, MICOL and GARCíA-BELLIDO 1990). Third, the phenotypes of heterozygotes involving recessive alleles and $U b x^{1}$ are indistinguishable from those involving other pseudopoint $U b x$ alleles which are known to produce abnormal UBX.

Pioneer observations of LEWIS (1951) (see results marked with a $c$ in Table 1) showed that some heterozygotes involving cis double mutants $b x U b x^{I}$ give stronger mutant phenotypes than those involving the single mutant $U b x^{l}$. These observations were then 
interpreted under the supposition that $b x$ and $U b x$ mutations affect two different genes, but can now be interpreted as suggesting the presence of remnant functions in $U b x^{1}$.

The behavior of $U b x^{1}$ is thus paradoxical because (1) it is amorphic as revealed by the phenotype of homozygotes and by its lack of detectable protein products, but (2) it is hypomorphic as revealed by the phenotype of its heterozygotes and by its sensitivity to additional mutations in the gene. Thus, $U b x^{l}$ behave as if it lacks morphogenetic products (proteins with "selector" activity, able to trans-regulate activity of other genes; GaRcí-BELlido 1975) but it retains regulatory function(s), that can affect the activity of a different allele of the $U b x$ gene in the homologous chromosome. Under this hypothesis, we have made a genetic study of those putative remnant functions of $U b x^{I}$ because (1) they could represent cis-regulatory functions involved in the normal control of expression of the $U b x$ gene, and (2) they could be involved in the trans interactions between homologous $U b x$ genes known as transvection effects (LewIS 1954). This study has been possible for $U b x^{l}$ because of the existence of double mutant recombinants involving this mutation and different recessive alleles of the $U b x$ gene, all of them obtained by E. B. LEwIs.

\section{MATERIALS AND METHODS}

Flies were cultured on standard medium under uncrowded conditions at $25 \pm 1^{\circ}$. All genetic variants used in this work have been previously described [LINDSLEY and GRELL (1968) and other references in the text]. In order to reduce the possible presence of spontaneous recessive modifiers, homozygous stocks were outcrossed to Vallecas wildtype strain. After two generations, homozygous flies were collected and used to do the crosses described in Tables 1 and 2.

\section{RESULTS}

The loss-of-function phenotype of the $U b x^{1}$ allele is exaggerated by additional cis recessive mutations: The fact that $U b x^{l}$ does not produce detectable UBX, but still shows less mutant phenotype over recessive alleles than a deficiency suggests that it retains some function(s) not mediated by UBX. We have tried to identify where these remnant functions are coded by studying the behavior of cis double mutant chromosomes carrying, in addition to $U b x^{l}$, one recessive mutation in the same gene. The mutant phenotypes have been scored in double heterozygotes with the same or other recessive alleles of the $U b x$ gene (Table 1). As controls we have analyzed the phenotypes of the different recessive alleles of the $a b x, b x, b x d$ and $p b x$ groups in heterozygotes with $U b x^{\prime}$ or $D f(3 R) P 9$, a deficiency for the entire $U b x$ gene (Figure 1). The recessive alleles are ordered in Table 1 after the location in the molecular map of their associated
TABLE 1

Metanotum and haltere mutant phenotypes of heterozygotes involving the $U b x^{\prime}$ allele

$U b x^{\prime} b x^{34 e} U b x^{l} b x^{3} U b x^{\prime} U b x^{\prime} b x d^{\prime} U b x^{\prime} p b x^{\prime} D f(3 R) P 9$

\begin{tabular}{|c|c|c|c|c|c|c|}
\hline \multicolumn{7}{|c|}{ Metanotum $^{a}$} \\
\hline \multicolumn{7}{|c|}{ bx phenotype } \\
\hline$a b x^{\prime}$ & $0-2$ & $0-2$ & $0-2$ & $0-2$ & $0-2$ & $2-4$ \\
\hline$a b x^{2}$ & $0-2$ & $0-2$ & $0-2$ & $0-2$ & $0-2$ & $2-3$ \\
\hline$a b x^{C A C: 4}$ & $0-2$ & $0-2$ & $0-2$ & $0-2$ & $0-2$ & 4 \\
\hline$b x^{F 3 l}$ & 0 & 1 & 1 & 0 & 0 & 1 \\
\hline$b x^{34 e}$ & $0^{c}$ & 3 & 3 & $3^{r}$ & 0 & 3 \\
\hline$b x^{34 e \cdot p r o s}$ & $0-2$ & $0-2$ & $0-2$ & $0-2$ & $0-2$ & $0-2$ \\
\hline$b x^{83 K a}$ & 1 & 4 & 4 & 4 & 2 & 4 \\
\hline$b x^{\prime}$ & $0^{r}$ & $0-1$ & $0-1^{\circ}$ & $0-1$ & 0 & $0-1$ \\
\hline$b x^{8}$ & 2 & 4 & 4 & 3 & 2 & 4 \\
\hline$b x^{3}$ & $\mathrm{l}^{\mathrm{c}}$ & 4 & 4 & $3^{r}$ & 1 & 4 \\
\hline$b x^{83 k b}$ & 1 & 4 & 4 & 3 & $\mathbf{l}$ & 4 \\
\hline \multicolumn{7}{|c|}{ pbx phenotype } \\
\hline$b x d^{55 i}$ & 2 & 2 & 2 & 4 & 2 & 2 \\
\hline$b x d^{\prime}$ & $2^{r}$ & 2 & 2 & $4^{r}$ & 2 & 2 \\
\hline$b x d^{5 / j}$ & 2 & 2 & 2 & 4 & 2 & 4 \\
\hline$b x d^{k}$ & $0-4$ & $0-4$ & ND & $0-4$ & 4 & 4 \\
\hline$p b x^{2}$ & 1 & 1 & 1 & 1 & 4 & 4 \\
\hline$p b x^{l}$ & 2 & 2 & 2 & 2 & 4 & 4 \\
\hline \multicolumn{7}{|l|}{ Haltere $^{b}$} \\
\hline \multicolumn{7}{|c|}{ bx phenotype } \\
\hline$a b x^{\prime}$ & $1-2$ & $0-1$ & $0-1$ & $1-2$ & $1-2$ & $3-4$ \\
\hline$a b x^{2}$ & $1-2$ & $0-1$ & $0-1$ & $1-2$ & $0-2$ & $3-4$ \\
\hline$a b x^{C A C \cdot 4}$ & 3 & $1-2$ & 1 & 3 & 3 & 4 \\
\hline$b x^{F 31}$ & 1 & 2 & 2 & 1 & 1 & 2 \\
\hline$b x^{34 y}$ & $1^{r}$ & 2 & 2 & $2^{r}$ & 1 & 2 \\
\hline$b x^{34 f-p r y}$ & $0-1$ & $0-1$ & $0-1$ & $0-1$ & $0-1$ & $0-1$ \\
\hline$b x^{83 K a}$ & 2 & 4 & 4 & 4 & 2 & 4 \\
\hline$b x^{l}$ & $0^{c}$ & 0 & $0-1^{r}$ & 0 & 0 & $0-1$ \\
\hline$b x^{8}$ & 3 & 4 & 4 & 4 & 3 & 4 \\
\hline$b x^{3}$ & $3^{r}$ & 4 & 4 & $4^{r}$ & 3 & 4 \\
\hline$b x^{83 K b}$ & 3 & 4 & 4 & 4 & 3 & 4 \\
\hline \multicolumn{7}{|c|}{ pbx phenotype } \\
\hline$b x d^{59 i}$ & 1 & 1 & 1 & 3 & 1 & 1 \\
\hline$b x d^{\prime}$ & 2 & 2 & 2 & $3^{c}$ & 3 & 2 \\
\hline$b x d^{5 l j}$ & 2 & 2 & 2 & 3 & 3 & 2 \\
\hline$b x d^{k}$ & $0-4$ & $0-4$ & ND & $0-4$ & 4 & 4 \\
\hline$p b x^{2}$ & 1 & 1 & 1 & 1 & 4 & 4 \\
\hline$p b x^{\prime}$ & 4 & 4 & 4 & 4 & 4 & 4 \\
\hline
\end{tabular}

Phenotypes corresponding to heterozygotes carrying one chromosome in the columns, the other in the rows were scored. Figures correspond to phenotypical classes ranging from 0 (wild type) to 4 (maximal mutant transformation encountered, usually corresponding to the almost complete homeotic transformation of the anlage). Variable expressivity of the mutant phenotype is represented by ranges between extreme values. At least 20 individuals of each genotype were studied. ND, phenotype not determined. For $a b x$ alleles, only the mutant phenotype affecting T3a has been studied, regardless of the mutant phenotype in T2p. For $b x d$ alleles, only the mutant transformation affecting $\mathrm{T} 3 \mathrm{p}$ has been studied, regardless of the mutant phenotype in Ala.

a Mutant phenotypes in metanotum (T3) were defined as follows: bx phenotype: transformation of anterior metanotum (T3a) toward anterior mesonotum (T2a); pbx phenotype: transformation of posterior metanotum (T3p) toward posterior mesonotum (T2p).

${ }^{b}$ Mutant phenotypes in haltere (T3) were defined as follows: bx phenotype: transformation of anterior haltere (T3a) toward anterior wing (T2a); pbx phenotype: transformation of posterior haltere (T3p) toward posterior wing (T2p). 1955)

Results similar to those previously published by LEwIS (1951, 

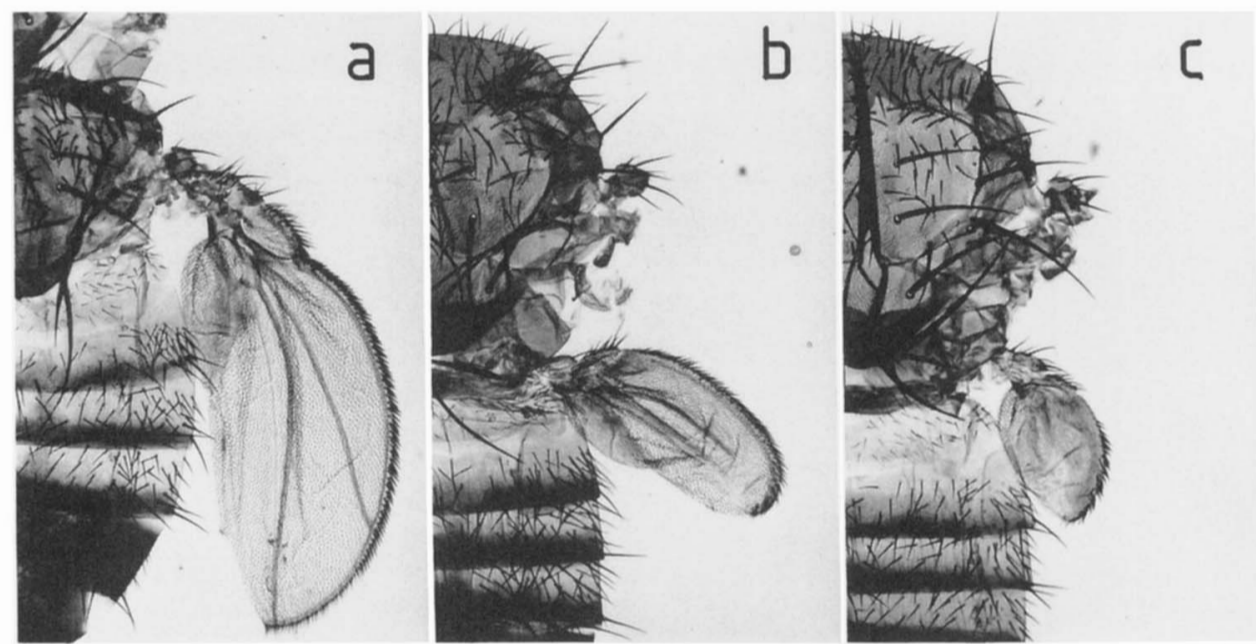

Figure 2.-Partial rescue of the bx phenotype of $a b x$ alleles by a $b x$ allele in cis to $U b x^{\prime}$. a) $D f(3 R) U b x 109 /$ $a b x^{C A C A}$; b) $U b x^{\prime} / a b x^{C A C A}$ and c) $b x^{3} U b x^{\prime} / a b x^{C A C 4}$. Wings were removed from the flies before mounting them. Note the different degrees of haltere (T3) toward wing (T2) transformations, that suggest more wild-type function in c than in b genotype. Both haltere toward wing and metanotum (T3) toward mesonotum (T2) transformations are almost complete in a, revealing the basal level of function of $a b x^{c A c t}$ allele. The partial rescues of the mutant phenotype in $b$ and $c$ must be due to the homologous chromosome. perturbations (Figure 1). The bx and pbx mutant phenotypes were studied both in metanotum and haltere (Table 1).

Our results in control genotypes agree with the conclusion of previous analyses in that for alleles within a group the bx mutant phenotype increases the closer is the perturbation to the promoter of the Ubx transcription unit, while the pbx mutant phenotype increases with the distance to that promoter (BENDER et al. 1983; PEIFER and BENDER 1986). Exceptions are $b x^{34 e-p r v}$ and $b x^{I}$ that have erratic phenotypes and lower expressivity than their neighboring alleles, perhaps because the molecular nature of the mutations (a "412" insert in the latter and a deletion in the former). The study of phenotypes of recessive alleles in heterozygotes with chromosomes carrying $U b x^{l}$ and a second recessive mutation uncovers qualitative differences between groups of alleles. All double mutant chromosomes show in certain heterozygotes with recessive alleles different phenotypes than those of the single mutant $U b x^{l}$ (Table 1 and Figures 2 and 3). Thus, $b x^{3} U b x^{l}$ or $b x^{34 e} U b x^{1}$ double mutant chromosomes cause stronger bx phenotypes over $b x$ alleles, but not stronger pbx phenotypes over $p b x$ and $b x d$ alleles. Interestingly, they reduce the bx haltere phenotypes in $a b x$ combinations (Figure 2; see DISCUSsion). The double mutant chromosome $U b x^{l} b x d^{l}$ shows stronger pbx phenotypes in heterozygotes with $b x d$ alleles, but also exaggerates the bx phenotype of $b x$ alleles (with the exceptions of $b x^{F 31}$ and $b x^{34 e-p r o}$, which have extremely variable phenotypes in heterozygotes with both $U b x^{l}$ and $\left.U b x^{I} b x d^{l}\right)$. $U b x^{l} p b x^{l}$ shows only stronger pbx phenotypes in heterozygotes with $p b x$ and $b x d$ alleles. The deletion of the whole $U b x$ gene $[D f(3 R) P 9]$ exaggerates the mutant phenotypes of all the recessive alleles with the exception of some bxd ones (see Discussion).

Recessive mutations cis-linked to $U b x^{I}$ differentially affect homologous allele expression depend- ing upon pairing conditions between homologous chromosomes: Phenotypic changes associated with variations in pairing conditions between homologous chromosomes have been called "transvection effects" (LEWIS 1954; see for reviews JudD 1988; Wu and GoldBERG 1989). LEwIS (1954) interpreted his observations on transvection effects as indicating that the interchange of gene products between homologs is facilitated or prevented depending on their degree of somatic pairing. In all the interactions showed in Table 1, we used structurally normal chromosomes allowing homologous Ubx chromosomal regions to pair in the interphase cellular nuclei. We have studied also the effect of perturbations in pairing between homologs on the modulation of the $b x^{34 e}$ allele by the remnant functions in $U b x^{l}$. The results of such experiments are shown in Table 2 and Figure 3. We have quantified the mutant phenotypes of individuals carrying $U b x^{l}, b x^{34 e}, b x d^{l}$ and two deficiencies for the entire $U b x$ gene $[D f(3 R) P 9$ (data not shown) and $D f(3 R) U b \times 109]$, in several cis and trans combinations using structurally normal chromosomes and four different rearranged $(R)$ chromosomes carrying breakpoints that presumably affect pairing of homologous Ubx gene regions (Figure 4). Heterozygotes between structurally normal chromosomes are expected to pair, allowing normal transvection to occur, giving phenotypes that we used as controls. Structural heterozygotes are expected to exaggerate the phenotype of the same genetic combinations due to hindrance in the transport of remnant functions. The extent of the mutant transformations was estimated by counting normal T2 structures that appear in mutant transformed T3 regions both in the metanotum and in the haltere (Table 2).

The most relevant results of Table 2, the metanotum (T3) toward mesonotum (T2) transformations, can be summarized as follows: (1) $b x^{34 e}$ homozygotes, whether associated or not with rearrangements [indi- 

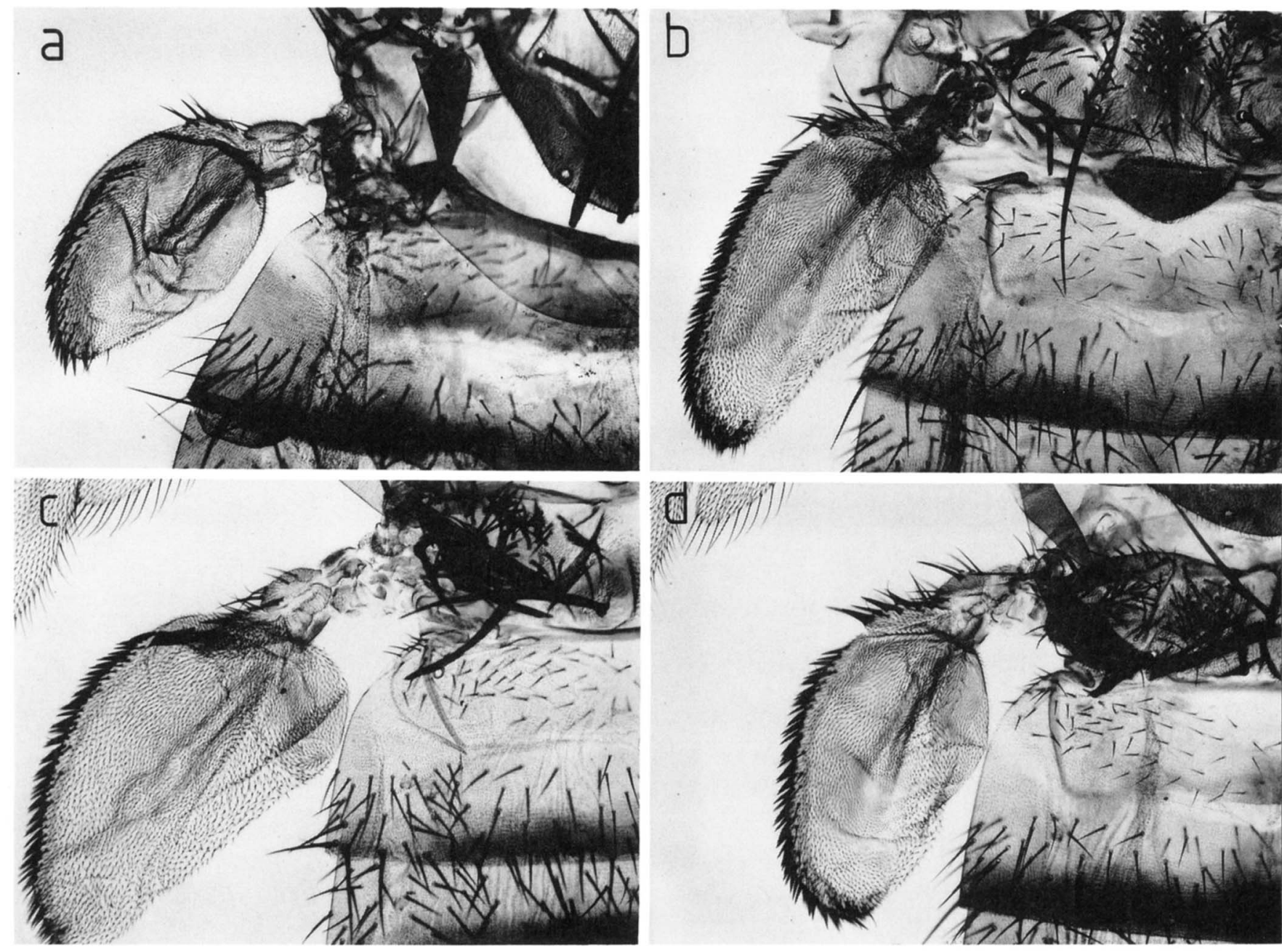

FigURE 3.-Effects of additional cis mutations on the phenotype of $U b x^{1} / b x^{3+e}$ genotype. a) $U b x^{l} / b x^{3+e}$; b) $D f(3 R) U b x 109 / b x^{3+t}$; c) $b x^{3} U b x^{1} /$ $b x^{3+r}$ and d) $U b x^{l} b x d^{l} / b x^{34 r}$. Notice the haltere (T3) toward wing (T2) and metanotum (T3) toward mesonotum (T2) homeotic transformations corresponding to variable depletion of remnant functions in the $U b x^{l}$ chromosome. Top right corner of a is occupied by a portion of normal mesonotum, being normal metanotum the region between that and first abdominal terguite (the region with short hairs). In $b, c$ and $d$ the same position show metanotum transformed toward mesonotum, being the normal mesonotum completely (b and c) or almost completely (d) out of the picture.

viduals of $b x^{34 e} / b x^{34 e}, b x^{34 e} / R\left(b x^{34 e}\right)$ or $R\left(b x^{34 e}\right) / R\left(b x^{34 e}\right)$ genotypes] show little differences among them, suggesting that the $b x^{34 e}$ allele behaves similarly irrespective of the structural nature of the chromosome in which it is located, and that the resulting phenotype is not dependent on putative spontaneous "modifier" mutations accumulated in the stocks and carried by the $R$ chromosomes involved. In all those genotypes, both homologous alleles are supposed to be producing UBX and consequently contributing to the adult phenotype. (2) $U b x^{I} / R\left(b x^{34 e}\right)$ individuals show stronger mutant phenotypes than $U b x^{1} / b x^{34 e}$ ones [in agreement with LEWIS (1954) and BABU and BHAT (1981)]. These results lead us to conclude, as did Lewis (1954), that the differences between a given $R\left(b x^{34 e}\right)$ and $b x^{34 e}$ chromosomes are due to disruption of pairing. In these genotypes only the $b x^{34 e}$ allele produces UBX, the expression of which is enhanced by the proximity of the homologous $U b x^{I}$ allele. It must be noted that there are clear differences between the three rearranged chromosomes in the same test; i.e., not all rearrangements enhance the bx mutant phenotype to the same extent. This suggests that disruption of the transvection phenomenon is not an "all or nothing," but a quantitative response. (3) It has been previously shown (Table 1) that $U b x^{l} b x d^{l} / b x^{34 e}$ flies have a bx phenotype stronger than that of $U b x^{1} / b x^{34 e}$ when both homologs are structurally normal and presumably paired. It also holds that $U b x^{l} b x d^{l} / R\left(b x^{34 e}\right)$ heterozygous have mutant phenotypes stronger than the corresponding $U b x^{1} / R\left(b x^{34 e}\right)$ ones, and the same is observed when comparing a given $b x^{34 e} U b x^{1} / R\left(b x^{34 e}\right)$ with the corresponding $U b x^{1} / R\left(b x^{34 e}\right)$. Since in the rearranged genotypes homologous $U b x$ gene regions are thought to be unpaired, clearly a second mutational damage in the $U b x^{I}$ chromosome (either $b x^{34 e}$ or $b x d^{l}$ ) 


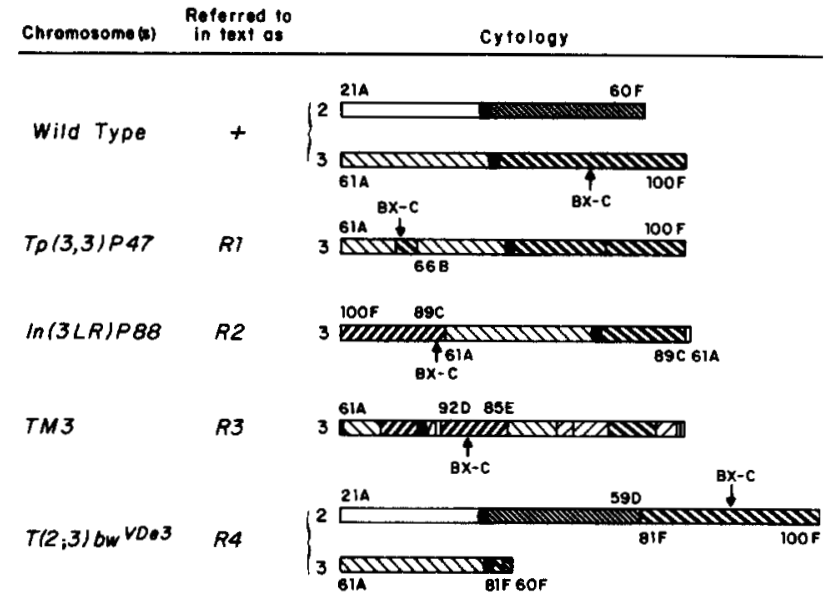

Figure 4.-Structure of rearranged chromosomes used in this work. In the wild-type chromosomes (top), each chromosomal arm is indicated by a different pattern, centromere as a black ellipse. Numbers on or under each chromosome indicate cytological map positions. Numbers just to the left of each chromosome indicate chromosome 2 or 3. The position of the bithorax complex (BX-C) is indicated by a vertical arrow. In the aberrant chromosomes, inverted patterns indicate chromosomal segments inverted in relation to their wild type orientation. The small black region in the left end of TM3 balancer chromosome is a translocation from the $X$ chromosome.

affects at a distance the expression of the $b x^{34 e}$ allele located in other chromosome.

As Table 2 also shows, the haltere toward wing transformation behaves in a different way: (1) It is less affected than the metanotum by the same genetic combinations, and (2) clear differences are observed only when comparing $b x^{34 e}$ homozygous (with or without rearrangements) with all the remaining genotypes. This different behavior of metanotal and haltere transformations may be indicative of different $U b x$ gene subfunctions controlling morphogenesis in different regions of the same imaginal disc.

Given the observation that the $b x d^{1}, b x^{3}$ and $b x^{34 e}$ mutations are associated with inserts of the gypsy transposable element (Figure 1), LEWIS (quoted in Babu, Selvakumar and Bhosekar 1987) suggested that the pairing of two adjacent gypsy inserts (for instance, in a $b x b \times d$ cis double mutant) could modify $U b x$ gene expression in a different way than that expected from additivity of single mutant phenotypes. If some of our results were due to pairing between transposons located in homologous paired chromosomes, so hindering $U b x$ gene transcription, the effect should be removed in conditions preventing pairing. Therefore we have compared several $U b x^{1} b x d^{1} / b x$ combinations with the corresponding $R\left(U b x^{l} b x d^{l}\right) / b x$ ones. In the case of $b x^{34 e}$ combinations (Table 2) we see that not only there is no reduction of the effect but a marked enhancement of the mutant phenotype. In other studied cases ( $b x^{3}$ and $b x^{83 K b}$, data not shown), the mutant phenotype remains the same, or is slightly
TABLE 2

Effects of rearrangements and additional mutations in the metanotum and haltere mutant phenotype of $b x^{34 c} / U b x^{l}$ heterozygous

\begin{tabular}{|c|c|c|c|c|}
\hline & $b x^{34 e}$ & $R 3\left(b x^{34 \prime}\right)$ & $R 2\left(b x^{346}\right)$ & $R I\left(b x^{34 y}\right)$ \\
\hline \multicolumn{5}{|l|}{ Metanotum } \\
\hline$b x^{34 e}$ & $42 \pm 5$ & & & \\
\hline$U b x^{\prime}$ & $0.6 \pm 1$ & $5 \pm 3$ & $20 \pm 6$ & $40 \pm 6$ \\
\hline$b x^{34 e} U b x^{l}$ & $71 \pm 1$ & $67 \pm 6$ & $68 \pm 5$ & $72 \pm 9$ \\
\hline$U b x^{\prime} b x d^{\prime}$ & $49 \pm 1$ & $44 \pm 5$ & $58 \pm 4$ & $56 \pm 6$ \\
\hline$R 4\left(U b x^{J} b x d^{l}\right)$ & $63 \pm 8$ & $55 \pm 6$ & $64 \pm 7$ & $64 \pm 6$ \\
\hline$R l\left(b x^{34 c}\right)$ & $42 \pm 7$ & $36 \pm 6$ & $44 \pm 6$ & Lethal \\
\hline$R 2\left(b x^{34 c}\right)$ & $40 \pm 8$ & $32 \pm 7$ & Lethal & \\
\hline$R 3\left(b x^{34 e}\right)$ & $39 \pm 8$ & Lethal & & \\
\hline$D f(3 R) U b \times 109^{a}$ & $66 \pm 8$ & $55 \pm 8$ & $76 \pm 9$ & $71 \pm 11$ \\
\hline \multicolumn{5}{|l|}{ Haltere } \\
\hline$b x^{34 e}$ & $34 \pm 6$ & & & \\
\hline$U b x^{\prime}$ & $50 \pm 5$ & $40 \pm 5$ & $44 \pm 4$ & $60 \pm 7$ \\
\hline$b x^{34} U b x^{l}$ & $58 \pm 5$ & $57 \pm 4$ & $59 \pm 5$ & $60 \pm 5$ \\
\hline$U b x^{\prime} b x d^{\prime}$ & $57 \pm 5$ & $54 \pm 4$ & $55 \pm 3$ & $62 \pm 6$ \\
\hline$R 4\left(U b x^{I} b x d^{l}\right)$ & $65 \pm 4$ & $64 \pm 5$ & $64 \pm 2$ & $62 \pm 5$ \\
\hline$R l\left(b x^{34 e}\right)$ & $26 \pm 6$ & $14 \pm 3$ & $24 \pm 4$ & Lethal \\
\hline$R 2\left(b x^{34 e}\right)$ & $29 \pm 4$ & $17 \pm 2$ & Lethal & \\
\hline$R 3\left(b x^{3+e}\right)$ & $25 \pm 5$ & Lethal & & \\
\hline$D f(3 R) U b \times 109^{a}$ & $57 \pm 4$ & $40 \pm 5$ & $56 \pm 6$ & $60 \pm 6$ \\
\hline
\end{tabular}

Phenotypes of heterozygotes for chromosomes on the horizontal and vertical axes were scored. Figures indicate T2 structural elements ( \pm standard deviation) present in mutant, partially transformed T3: microchaete by hemimetanotum (normal metanotum has no microchaete) or medial triple row elements per haltere (normal haltere has no medial triple row elements) for at least 10 individuals of each sex. Structures of $R 1$ to $R 4$ chromosomes are shown in Figure 4.

${ }^{a}$ Similar results were obtained with $D f(3 R) P 9$

enhanced, in $R\left(U b x^{I} b x d^{I}\right) / b x$ flies compared with the $U b x^{l} b x d^{l} / b x$ ones. These results make it improbable that a trans interaction between neighbor (paired) gypsy inserts accounts for our observations. Furthermore, the $U b x^{I} b x d^{l}$ chromosome affects also the phenotypes of non-gypsy $b x$ alleles, for example $b x^{8}$ and $b x^{I}$ (Table 1). In addition, the phenotypes of $U b x^{I} b x d^{I} /$ $b x d$ individuals are the same in paired and unpaired conditions (data not shown).

\section{DISCUSSION}

The $U b x^{1}$ allele retains regulatory functions affecting homologous $U \boldsymbol{b x}$ gene activity: As Table 1 shows, the mutant phenotypes of trans-heterozygotes involving $U b x^{1}$ and a given recessive allele of the $U b x$ gene can be modified by additional mutations in the $U b x^{I}$ chromosome. The expected noncomplementing phenotypes are obtained when the recessive mutations are of the same allelic type $(b x, b x d$ and $p b x)$ in both homologous chromosomes. This strongly suggests that the $U b x^{l}$ allele retains remnant functions that can be depleted by mutational damages in both the Ubx transcription unit introns and in the bxd region of the gene. Those remnant functions in the $U b x^{l}$ chromosome are more effectively depleted or abolished by 
the $b x d^{I}$ mutation than by any other of the mutations studied here. As the $U b x^{1}$ allele is unable to produce protein products, those variations in the mutant phenotypes must originate from variations in the production of UBX by the homologous recessive allele, the activity of which is modulated by remnant regulatory functions in $U b x^{l}$.

$U b x^{1}$ remnant functions differentially modulate homologous allele expression depending upon pairing conditions between homologous chromosomes: As seen in Table 2 the phenotypes of combinations involving the $b x^{34 e}$ allele differ depending on the presence or not of a second mutation in the $U b x^{I}$ allele and the chromosome rearrangement used. $b x^{34 e}$ homozygotes give similar phenotypes, whether or not they are associated with rearrangements, as expected since both homologs are affected in the same functions. The weakest mutant phenotype corresponds to $b x^{34 e} /$ $U b x^{l}$ heterozygous, the strongest to those of $b x^{34 e} /$ $D f(3 R) U b \times 109$. While in the latter genotype the production of UBX by the $b x^{34 e}$ allele is exclusively modulated by its own cis-regulatory elements, $b x^{34} / U b x^{1}$ individuals show the maximum effect of the regulatory functions of $U b x^{l}$ enhancing the expression of UBX by $b x^{34 e}$ allele. The $b x^{34 e} U b x^{1}$ chromosome gives similar phenotypes to those of $D f(3 R) U b \times 109$, suggesting that the $b x^{34 e}$ mutation abolishes the remnant regulatory functions in $U b x^{I}$. Combinations of any $R\left(b x^{34 e}\right)$ and either $U b x^{1}$ or $U b x^{I} b x d^{l}$ yield intermediate phenotypes that must correspond to "degrees" in transvection. When structural heterozygotes (genotypes involving $R$ chromosomes) are compared, the extent of the mutant phenotype follows the order $R 3$ $<R 2<R 1$. These results taken as a whole confirm the conclusions obtained from the behavior of paired chromosomes (Table 1) that the signals arising from $U b x^{I}$ act more efficiently than those from $b x^{34} U b x^{I}$ or $U b x^{I} b x d^{I}$ chromosomes. They show in addition (1) that the effectiveness of those signals on the activity of the homologous allele varies with the $R$ chromosome used, suggesting that the signals reach the homolog depending on the degree of pairing or proximity between homologous copies of the $U b x$ gene in different rearrangements, and (2) that additional mutations in a $U b x^{l}$ chromosome are perceived by the homolog even in conditions of unpairing.

Several $U b x$ cis-regulatory elements are able to act in trans: At least two different cis-regulatory elements of the $U b x$ gene seem to be able to modulate in trans the expression of the homologous allele. One is damaged by $b x^{34 e}$ and $b x^{3}$ mutations and positively controls $U b x$ expression exclusively in T3a. The other is damaged by the $p b x^{l}$ mutation and positively controls $U b x$ expression exclusively in T3p.

Some other regulatory elements might be inferred from unexpected phenotypes obtained in certain com- binations. First, the presence of $b x d^{1}$ in the $U b x^{1}$ chromosome exaggerates the phenotype of most $b x$ alleles, confirming an observation of LEWIS (1955). Second, the pbx phenotype is stronger in $U b x^{l} b x d^{l} / b x d$ heterozygotes than in $D f(3 R) P 9 / b x d$ ones, although this deficiency entirely lacks the $b x d$ region. Third, for all the $a b x$ alleles tested, $b x^{34} U b x^{1} / a b x$ and $b x^{3} U b x^{1} / a b x$ show partial rescue of the bx mutant phenotype compared to $U b x^{l} / a b x$ individuals. These findings confirm that mutations in both the introns of the Ubx transcription unit and the bxd region affect different signals present in, or arising from, the $U b x^{l}$ chromosome that modulate the expression of the homologous $U b x$ gene.

It is generally assumed that regulatory functions in the bxd region modulate $U b x$ gene activity exclusively in T3p and A la, and those of abx and bx region the activity of the gene in T3a (DUNCAN 1987; AKAM, DAwson and TEAR 1988). Therefore, it is remarkable that the $b x d^{l}$ mutation affects the T3a mutant phenotype of $b x$ alleles, which suggests the presence in the bxd region of at least one regulatory element affecting gene activity in T3a. As Table 1 shows, the effects of the $U b x^{l} b x d^{l}$ chromosome on bx phenotypes are almost of the same extent as those caused by $b x^{3} U b x^{1}$ and $b x^{34 e} U b x^{1}$ chromosomes upon the same $b x$ alleles. However, the behavior of the $U b x^{1} b x d^{1}$ chromosome differs from those of $b x^{3} U b x^{1}$ or $b x^{34 e} U b x^{1}$ in that (a) the former leave apparently unaffected the bx phenotype of $a b x$ alleles, while the two latter chromosomes rescue all the studied bx haltere phenotypes of $a b x$ alleles, and (b) the latter do not affect the pbx phenotypes of $b x d$ alleles. This differential behavior suggests that the $U b x^{l}$ chromosome retains at least two signals, one in the bx and the other in the bxd region, that interact with themselves and with the homologous $U b x$ gene (depending on which regions remain functional in it) to give an outcome of more or less gene activity as measured in the adult phenotype. In this context it is relevant that the effects of a $U b x^{l} b x d^{l}$ chromosome upon the pbx phenotype of $b x d$ alleles are stronger than those of a deficiency for the whole gene, $D f(3 R) P 9$ (Table 1). This could be explained by the existence of some negative regulatory element in the bxd region which is not damaged by $b x d^{I}$ and which enhances the insufficiency associated with the positive element(s) of control damaged by the mutation. The absence of both positive and negative elements (in the heterozygote involving a deficiency for the gene) might allow for a more compensated expression of the homolog.

The phenotypes of $b x U b x^{I} / a b x$ individuals (Figure 2) lead to a paradox: one loss-of-function mutation $(b x)$ in the $U b x$ gene partially rescues the phenotype of another loss-of-function mutation $(a b x)$ in the homologous $U b x$ gene. On the basis of the phenotypes 
of trans-heterozygotes between different breakpoints affecting the Ubx transcription unit and recessive $b x$ and $a b x$ alleles, АкAM et al. (1985) have suggested the existence of a yet-to-be-discovered transcription unit, overlapping with the $3^{\prime}$-most region of the Ubx transcription unit. In addition, LEWIS (1985) has described a phenomenon termed cis-overexpression, by which loss-of-function mutations in the $U b x$ gene (i.e., a given $b x$ allele) behave as producing a gain of function in the immediately adjacent, most proximal "unit of function" in the gene (i.e, $a b x)$. Our observations on $b x U b x^{1} / a b x$ phenotypes, which involve interactions between units of function located in homologous genes, are similar to those of LewIs, which involve interactions between units of function located in cis in the same gene. Taking all these data together, one may speculate about the existence of the new transcription unit proposed by Акам et al.: the presence of a transcriptionally active gypsy insert (as $b x^{34 e}$ and $b x^{3}$ ) located in the neighborhood of the promoter of that transcription unit could be the cause of the "cisoverexpression" observed by LEwIs. The enhanced production of that RNA product in a $b x^{3} U b x^{1}$ or a $b x^{34 e} U b x^{I}$ chromosome would result in the partial rescue of the loss of function of an $a b x$ homolog.

Implications for transvection models: Two main hypotheses have been proposed to explain the transvection phenomenon: (1) the interaction between a regulatory element in one gene and the promoter of the homologous allele (ZACHAR, Chapman and Bingham 1985; Pirrotta, Steller and Bozzetti 1985), mediated by enhancer-binding proteins and the formation of large, interchromosomal DNA loops (BENSON and PIRrotTA 1988) and (2) the involvement of diffusible transcriptional factors, with differential distribution inside the cellular nucleus (LEwIS 1985; KORNHER and BrUTlag 1986). Some authors have speculated about these transcription factors being short-radius-of-action regulatory RNAs transcribed from the genes involved in the transvection phenomenon (JACK and JUDD 1979; MICOL and GARCía-BELLIDO 1988; CASTElli-Gair, Micol and García-BeLLIDO 1990; D. MATHOG, manuscript in preparation). However, all these models are, at present, purely conjectural due to the lack of molecular evidence.

Needless to say, from our present genetic results and using the available molecular data, but in the absence of a molecular analysis of the problem, it is not possible to demonstrate conclusively the molecular nature of the transvecting signals emanating from the $U b x^{l}$ allele. However, some inferences and proposals can be discussed. On the basis of the results presented in Table 1 we cannot distinguish between short-radius-of-action gene products such as RNAs, diffusing from $U b x^{1}$ to its homolog, or enhancer sequences acting in trans when the homologs are syn- apsed. These alternatives can be discussed using the information provided by changing the pairing conditions of the homologs (Table 2). Genetic and molecular studies led to the suggestion of chromosome to chromosome interactions involving physical contact between regulatory DNA sequences mediated by the DNA-binding products of the zeste gene (reviewed in Wu and GolDBERG 1989). This hypothesis is applicable to our results, but it should explain (1) the way in which interaction between enhancers and promoters could result in the degrees of transvection observed, and (2) the perception by $b x^{34 e}$ allele of a second mutational damage in a $U b x^{l}$ chromosome in conditions in which homologous Ubx chromosomal regions are unpaired. Both the quantitative nature of the transvection phenomenon and the perception at a distance of mutational damage in the homolog shown in Table 2 could be explained in terms of regulatory RNA products of the $U b x$ gene, with an effectiveness progressively reduced with the distance to the site of production. Although regulatory functions were proposed as a possible role for RNAs from the bxd units (HOGNESS et al. 1985), this alternative encounters the difficulty that no RNAs arising from the bxd region have been found in metathoracic discs during larval development (Lipshitz, Peattie and Hogness 1987), when the mutant phenotypes are generated. Furthermore, there is no molecular evidence of RNAs as transcriptional activators, and only a few cases of autogenous regulation of gene activity by RNAs are known, all of them being instances of RNAs as negative regulators (ОкамOTO and FreUnDLICH 1987).

Thanks are due to E. B. Lewis, G. Morata and W. BENDER for providing stocks, to M. P. CAPDEVILA for discussions, and to F. J. Calzone, E. H. Davidson, E. B. Lewis, D. R. Mathog, E. M. MEYEROWITZ and J. E. MiNOR, JR., for comments on the manuscript. This work was supported by Comisión Asesora de Investigación Científica y Técnica, Comisión Interministerial de Ciencia y Tecnologia, Fundación Ramón Areces and Fundación Juan March. J.L.M. was postdoctoral fellow of Fundación Juan March. J.E.C.-G. was fellow of British Council-Consejo Superior de Investigaciones Cientificas.

\section{LITERATURE CITED}

Akam, M. E., I. Dawson and G. Tear, 1988 Homeotic genes and the control of segment diversity. Development 104 (Suppl.): 123-133.

Akam, M. E., A. Martínez-Arias, R. Weinzierl and C. D. Wilde, 1985 Function and expression of Ultrabithorax in the Drosophila embryo. Cold Spring Harbor Symp. Quant. Biol. 50: 195-200.

BABU, P., and S. G. BHAT, 1981 Role of zeste in transvection at the bithorax locus of Drosophila. Mol. Gen. Genet. 183: 400402.

Babu, P., K. S. Selvakumar and S. Bhosekar, 1987 Studies on transvection at the bithorax complex in Drosophila melanogaster. Mol. Gen. Genet. 210: 557-563.

BeACHY, P. A., S. L. Helfand and D. S. Hogness, 1985 Segmental distribution of bithorax complex proteins during Drosophila development. Nature 313: 545-551. 
Bender, W., M. Akam, F. Karch, P. A. Beachy, M. Peifer, P. SPIERER, E. B. LewiS and D. S. Hogness, 1983 Molecular genetics of the bithorax complex in Drosophila melanogaster. Science 221: 23-29.

Bender, W., B., Weiffenbach, F. Karch and M. Peifer, 1985 Domains of cis-interaction in the bithorax complex. Cold Spring Harbor Symp. Quant. Biol. 50: 173-180.

Benson, M., and V. PirrotTa, 1988 The Drosophila zeste protein binds cooperatively to sites in many gene regulatory regions: implications for transvection and gene regulation. EMBO J. 7: 3907-3915.

Botas, J., C. V. Cabrera and A. García-Bellido, 1988 The reinforcement-extinction process of selector gene activity: a positive feed-back loop and cell-cell interactions. Wilhelm Roux's Arch. Dev. Biol. 197: 424-434.

Cabrera, C. V., J. Botas and A. García-Bellido, 1985 Distribution of proteins in mutants of bithorax complex genes and its transregulatory genes. Nature 318 : 569-571.

CaPdevit.A, M. P., and A. García-Bellido, 1974 Development and genetic analysis of bithorax phenocopies in Drosophila. Nature 250: 500-502.

Capdevila, M. P., and A. García-Bellido, 1978 Phenocopies of bithorax mutants. Genetic and developmental analyses. Wilhelm Roux's Arch. Dev. Biol. 185: 105-126.

Capdevila, M. P., and A. García-Bellido, 1981 Genes involved in the activation of the bithorax complex of Drosophila. Wilhelm Roux's Arch. Dev. Biol. 190: 339-350.

Castelli-Gair, J.E., J. L. Micol and A. García-Bellido, 1990 Transvection in the Drosophila Ultrabithorax gene: a $C b x^{I}$ mutant allele induces ectopic expression of a normal allele in trans. Genetics 126: 177-184.

DunCan, I., 1987 The bithorax complex. Annu. Rev. Genet. 21: 285-319.

Garcí-Bellido, A., 1975 Genetic control of wing disc development in Drosophila, pp. 161-182 in Cell Patterning (Ciba Symposium 29), edited by S. Brenner. Elsevier, Amsterdam.

GonzÁlez-Gaitán, M. A., J. L. Micol and A. García-Bellido, 1990 Developmental genetic analysis of Contrabithorax mutations in Drosophila melanogaster. Genetics 126: 139-155.

Hayes, P. H., T. Sato and R. E. Denell, 1984 Homoeosis in Drosophila: the Ultrabithorax larval syndrome. Proc. Natl. Acad. Sci. USA 81: 545-549.

hogness, D. S., H. D. Lipshitz, P. A. Beachy, D. A. Peattie, R. B. Saint, M. Goldschmidt-Clermont, P. J. Harte, E. R. Gavis and S. L. HeLfand, 1985 Regulation and products of the Ubx domain of the bithorax complex. Cold Spring Harbor Symp. Quant. Biol. 50: 181-194.

INGHAM, P. H., 1985 Genetic control of the spatial pattern of selector gene expression in Drosophila. Cold Spring Harbor Symp. Quant. Biol. 50: 201-208.

JACK, J. W., and B. H. JudD, 1979 Allelic pairing and gene regulation: a model for the zeste-white interaction in Drosophila melanogaster. Proc. Natl. Acad. Sci. USA 76: 1368-1372.

JUDD, B. H., 1988 Transvection: allelic cross talk. Cell 53: 841843.

Kerridge, S., and G. Morata, 1982 Developmental effects of some newly induced Ultrabithorax alleles of Drosophila. J. Embryol. Exp. Morphol. 68: 211-234.

Kornher, J. S., and D. BrutlaG, 1986 Proximity-dependent enhancement of $\mathrm{Sgs}_{s-4}$ gene expression in D. melanogaster. Cell 44: $879-883$

LEwrs, E. B., 1951 Pseudoallelism and gene evolution. Cold Spring Harbor Symp. Quant. Biol. 16: 159-174.

LEwIS, E. B., 1954 The theory and application of a new method of detecting chromosomal rearrangements in Drosophila melanogaster. Am. Nat. 88: 225-239.
LEWrs, E. B., 1955 Some aspects of position pseudoallelism. Am. Nat. 89: 73-89.

LEWIS, E. B., 1963 Genes and developmental pathways. Am. Zool. 3: 33-56.

LEwis, E. B., 1978 A gene complex controlling segmentation in Drosophila. Nature 276: 565-570.

LEWIS, E. B., 1985 Regulation of the genes of the bithorax complex in Drosophila. Cold Spring Harbor Symp. Quant. Biol. 50: $159-174$.

LINDSLeY, D. L., and E. H. Grell, 1968 Genetic Variations of Drosophila melanogaster. Carnegie Inst. Wash. Publ. 627.

Lifshitz H. D., D. A. Peattie and D. S. Hogness, 1987 Novel transcripts from the Ultrabithorax domain of the bithorax complex. Genes Dev. 1: 307-322.

MAHAFFEY, J. W., and T. C. KaUfMaN, 1988 The homeotic genes of the Antennapedia complex and the bithorax complex of Drosophila, pp. 329-360 in Developmental Genetics of Higher Organisms: A Primer in Developmental Biology, edited by G. M. MaLACINSKI. Macmillan, New York.

Micol, J. L., and A. García-Bellido, 1988 Genetic analysis of "transvection" effects involving Contrabithorax mutations in Drosophila melanogaster. Proc. Natl. Acad. Sci. USA 85: 11461150.

Miñana, F. J., and A. Garcia-Belliroo, 1982 Preblastoderm mosaics of mutants of the bithorax-complex. Wilhelm Roux's Arch. Dev. Biol. 191: 331-334.

Morata, G., and A. Garcia-Bellido, 1976 Developmental analysis of some mutants of the bithorax system of Drosophila. Wilhelm Roux's Arch. Dev. Biol. 179: 125-146.

Morata, G., and S. KerRidge, 1980 An analysis of the expressivity of some bithorax transformations, pp. 141-154 in Development and Neurobiology of Drosophila, edited by O. SIDIQQI, P. Babu, L. Hall and J. Hall. Plenum Press, New York.

Morata, G., E. Sánchez-Herrero and J. Casanova, 1986 The bithorax complex of Drosophila: an overview. Cell Differ. 18: $67-78$.

O'Connor, M. B., R. Binari, L. A. Perkins and W. Bender, 1988 Alternative RNA products from the Ultrabithorax domain of the bithorax complex. EMBO J. 7: 435-445.

OKAMOTO, K., and M. FREUNDLICH, 1987 Autogenous regulation of transcription of the $\operatorname{crp}$ operon by a divergent RNA transcript, pp. 357-369 in Molecular Biology of RNA: New Perspectives, edited by M. INOUYE and B. S. Dudock. Academic Press, San Diego.

PeIfer, M., and W. BENDER, 1986 The anterobithorax and bithorax mutations of the bithorax complex. EMBO J. 5: 2293-2303.

PeIfer, M., F. KARCH and W. BENDER, 1987 The bithorax complex: control of segmental identity. Genes Dev. 1: 891-898.

Pirrotta, V., H. Steller and M. P. Bozzetti, 1985 Multiple upstream regulatory elements control the expression of the Drosophila white gene. EMBO J. 4: 3501-3508.

Sánchez-Herrero, E., J. Casanova and G. Morata, 1988 Genetic structure of the bithorax complex. BioEssays 8: 124-127.

Weinzierl, R., J. M. Axton, A. Ghysen and M. AKam, 1987 Ultrabithorax mutations in constant and variable regions of the protein coding sequence. Genes Dev. 1: 386-397.

White, R. A. H., and M. Wilcox, 1984 Protein products of the bithorax complex in Drosophila. Cell 39: 163-171.

WHITE, R. A. H., and M. WILcox, 1985 Regulation of the distribution of Ultrabithorax proteins in Drosophila. Nature 318: 563-567.

Wu, C.-T., and M. L. GoldBerg, 1989 The Drosophila zeste gene and transvection. Trends Genet. 5: 189-194.

ZaChar, S., C. H. Chapman and P. M. Bingham, 1985 On the molecular basis of transvection effects and the regulation of transcription. Cold Spring Harbor Symp. Quant. Biol. 50: 337346.

Communicating editor: P. CherRas 